\title{
Analysis and effect of conventional flasks in shaking culture of Escherichia coli
}

\author{
Masato Takahashi and Hideki Aoyagi* (1)
}

\begin{abstract}
The circulation direct monitoring and sampling system (CDMSS) is used as a monitoring device for $\mathrm{CO}_{2}$ and $\mathrm{O}_{2}$ concentrations of bypass type in shake-culture flask. The CDMSS could measure $k_{\perp} a$, an index for evaluating the performance of aerobic culture incubators, and $k_{\mathrm{G}}$ an indicator of the degree of $\mathrm{CO}_{2}$ ventilation in the flask gas phase. We observed that cylindrical flasks provided a different culture environment, yielded a much higher $k_{\mathrm{G}}$ than the Erlenmeyer and Sakaguchi flasks, and yielded $k_{\perp}$ a equivalent to that by Erlenmeyer flask by setting the ring-type baffle appropriately. Baffled cylindrical flask used for Escherichia coli K12 IFO3301 shake culture maintained lower $\mathrm{CO}_{2}$ concentrations in the headspace than conventional flasks; therefore, $\mathrm{CO}_{2}$ accumulation in the culture broth could be suppressed. Cell growth in baffled cylindrical flask (with $k_{\perp}$ a equivalent to that of the Erlenmeyer flask) was about 1.3 and 1.4 times that in the Erlenmeyer and Sakaguchi flasks, respectively. This study focused on the batch culture at the flask scale and designed the headspace environment with low $\mathrm{CO}_{2}$ accumulation. Therefore, we conclude that redesign of flasks based on $k_{\mathrm{L}}$ a and $k_{\mathrm{G}}$ may contribute to a wide range of fields employing microorganism culture.
\end{abstract}

Keywords: Aerobic culture, Carbon dioxide, Escherichia coli, Headspace, Monitoring device, Shake-flask culture

\section{Introduction}

In 1932, shake-flask culture was developed for submerged culture of fungi to overcome the biomass yield limitations of surface culture (Kluyver et al. 1933). Erlenmeyer flask is now widely used to culture microorganisms and plant and animal cells (Weirether et al. 1968; Klöckner and Büchs 2012; Platas et al. 2013; Reynoso-Cereceda et al. 2016; Eibl et al. 2018). Shake-flask culture of microorganisms is aerobic and aims to provide sufficient stirring and oxygen to the culture broth and enables microorganisms to be batch cultured in parallel at low cost. Therefore, shake-flask culture is frequently used to screen secondary metabolites and to optimise culture conditions for microorganisms in the initial steps of bioprocess development. However, unlike in the case of agitated bioreactors (such as jar fermenters), the various culture conditions in shake

*Correspondence: aoyagi.hideki.ge@u.tsukuba.ac.jp

Faculty of Life and Environmental Sciences, University of Tsukuba,

Tsukuba, Ibaraki 305-8572, Japan flasks generally cannot be monitored without dedicated devices.

Monitoring technology in shake flasks has been becoming popular. Gaseous monitoring devices are remarkable because accurate measurement with conventional sampling methods, which include the interruption of shake culture and the transporting to a clean bench, the opening of culture plug at clean bench, are difficult to achieve due to Henry's law (Takahashi et al. 2017; Takahashi and Aoyagi 2018a). The methods of monitoring gas concentration in shake-flask culture are characterised by the measuring site, the principle of measurement equipment, and the measurement style (Takahashi and Aoyagi 2018b). The standard direct device is the Respiration Activity Monitoring System (RAMOS), which can monitor the flask gas phase (Anderlei et al. 2004). To the best of our knowledge, the Circulation Direct Monitoring and Sampling System (CDMSS) is the first bypass device to be developed that can monitor the behaviour of $\mathrm{CO}_{2}$ and $\mathrm{O}_{2}$ in the gas-liquid phases and obtain a sample without interrupting the shaking of the culture (Takahashi
Springer Open (c) The Author(s) 2020. This article is licensed under a Creative Commons Attribution 4.0 International License, which permits use, sharing, adaptation, distribution and reproduction in any medium or format, as long as you give appropriate credit to the original author(s) and the source, provide a link to the Creative Commons licence, and indicate if changes were made. The images or other third party material in this article are included in the article's Creative Commons licence, unless indicated otherwise in a credit line to the material. If material is not included in the article's Creative Commons licence and your intended use is not permitted by statutory regulation or exceeds the permitted use, you will need to obtain permission directly from the copyright holder. To view a copy of this licence, visit http://creativeco mmons.org/licenses/by/4.0/. 
et al. 2017). A device that combines the direct type and the bypass type has also been recently reported (Schulte et al. 2018). Most monitoring devices for shake-flask cultures remain only an implementation to Erlenmeyer flask (Anderlei et al. 2004; Ge and Rao 2012; Takahashi et al. 2017). Alternatively, several studies have focused on the shake culture compatibility between an Erlenmeyer flask and microplate, based on the same measurement principle of RAMOS (Wewetzer et al. 2015). On the contrary, in shake-flask culture, not only Erlenmeyer flasks, but also Sakaguchi flasks, are frequently used in reciprocal shaking (Shiota and Sakaguchi 1950; Omura et al. 1977; Hirasawa et al. 2006; Nojiri et al. 2015; Matsuda et al. 2017). There are few reports on monitoring of culture broth in Sakaguchi flask, and only one report on the headspace of Sakaguchi flask, which identified a concentration gradient in the vertical direction of the gas phase (Takahashi and Aoyagi 2018c). There is no research yet on monitoring and comparison of the $\mathrm{CO}_{2}$ and $\mathrm{O}_{2}$ concentrations in the headspace and culture broth in shake culture in conventional flasks, such as Erlenmeyer and Sakaguchi flasks. There are not sufficient studies on the relationship between flask shape and $\mathrm{CO}_{2}$ and $\mathrm{O}_{2}$ in shake-culture flasks of microorganisms.

Our study examined the behaviour of Escherichia coli K12 IFO3301 shake culture in various flasks by using circulation direct monitoring and sampling system (CDMSS). CDMSS was also used to calculate the total oxygen transfer capacity coefficient $\left(k_{\mathrm{L}} a\right)$, which is an indicator of the capacity to supply oxygen from the headspace to the liquid via the gas-liquid interface, and $k_{\mathrm{G}}$, which is an indicator of $\mathrm{CO}_{2}$ ventilation from the headspace through the breathable stopper into the atmosphere, in Erlenmeyer and Sakaguchi flasks. Based on these findings, we utilised and evaluated the flasks of cylindrical shape with $\mathrm{CO}_{2}$ ventilation capacity in the headspace, which cannot be obtained in Erlenmeyer and Sakaguchi flasks.

\section{Materials and methods}

Microorganisms, medium, and inoculum preparation

Escherichia coli K12 IFO3301 was selected as the experimental organism. The LB medium ( $\mathrm{pH} 7.0)$ used to culture E. coli $\mathrm{K} 12$ IFO3301 consisted of: (in g/L) tryptone, 10; yeast extract, 5; and $\mathrm{NaCl}, 5$. A loop-full of $E$. coli K12 IFO3301 slant culture was inoculated into a 500$\mathrm{mL}$ Erlenmeyer flask containing $100 \mathrm{~mL}$ of LB medium. The sample was then cultured at $30{ }^{\circ} \mathrm{C}$ on a rotary shaker with $70 \mathrm{~mm}$ shaking diameter at $200 \mathrm{rpm}$ for $7.5 \mathrm{~h}$. Glycerol stocks were prepared by adding the culture medium to glycerol (final glycerol concentration: $20 \%[\mathrm{v} / \mathrm{v}]$ ) and stored at $-80^{\circ} \mathrm{C}$.

\section{Culture conditions}

Erlenmeyer, Sakaguchi, cylindrical, and baffled cylindrical flasks were selected for the study. All flasks were $500-\mathrm{mL}$ in size. A detachable, O-ring shaped baffle was selected for constructing the baffled cylindrical flask (6 $\mathrm{cm}$ from the bottom). One $\mathrm{mL}$ each of glycerol stock was inoculated into a 500-mL Erlenmeyer flask and a 500$\mathrm{mL}$ cylindrical flask containing $100 \mathrm{~mL}$ of LB medium, respectively, and cultured at $30{ }^{\circ} \mathrm{C}$ on a rotary shaker with $70 \mathrm{~mm}$ shaking diameter at $200 \mathrm{rpm}$. In the case of the $500-\mathrm{mL}$ Sakaguchi flask containing $100 \mathrm{~mL}$ of LB medium, $1 \mathrm{~mL}$ of glycerol stock was inoculated and cultured at $30{ }^{\circ} \mathrm{C}$ on a reciprocating shaker with $70 \mathrm{~mm}$ shaking diameter at 120 strokes/min. Lastly, $50 \mathrm{~mL}$ of LB medium was added to the $500-\mathrm{mL}$ baffled cylindrical flask and then $0.5 \mathrm{~mL}$ of glycerol stock was inoculated, cultured in the same way as Erlenmeyer flask and cylindrical flask. The shaking conditions of the Erlenmeyer flask and the Sakaguchi flask were those of the most frequently used rotary type and reciprocating type, respectively, which are standard culture conditions of $E$. coli.

\section{Measurement of $k_{\mathrm{L}} \mathrm{a}$}

Dissolved oxygen concentration was measured with the CDMSS using the sulfite oxidation method, and the total $\mathrm{O}_{2}$ transfer capacity coefficient $\left(k_{\mathrm{L}} a\right)$ was calculated as follows:

$$
-\ln \frac{C_{\max }-C}{C_{\max }-C_{0}}=k_{L} a \cdot t
$$

where $t$ is time (s), $\mathrm{C}$ is the measurement value of dissolved oxygen concentration, $\mathrm{C}_{0}$ is the dissolved oxygen concentration at $t=0(\mathrm{mg} / \mathrm{L})$, and $\mathrm{C}_{\max }$ is the maximum dissolved oxygen concentration under the above-mentioned conditions. The maximum dissolved oxygen concentration was determined just before the measurements were taken for all conditions. When the decision coefficient value (termed $R^{2}$ ) of the approximate expression of Eq. 1 exceeded 0.90, the slope of the graph was set as $k_{\mathrm{L}} a$.

Preliminary experiments confirmed that the $\mathrm{O}_{2}$ concentration in the headspace of all the flasks decreased during measurement. The headspace was continuously suctioned $(200 \mathrm{~mL} / \mathrm{min})$ by using the CDMSS and the suctioned gas was exhausted without circulation to the headspace to prevent a decrease in the $\mathrm{O}_{2}$ concentration. In all the experiments for $k_{\mathrm{L}} a$ determination, dissolved oxygen was measured while confirming that the $\mathrm{O}_{2}$ concentration in the headspace was equivalent to that in the atmosphere. The shaking conditions were constant at 200 rpm for the Erlenmeyer and cylindrical flasks and 120 strokes/min for the Sakaguchi flask. The working volume was set to 100 or $50 \mathrm{~mL}$, and the $k_{\mathrm{L}} a$ for each condition was calculated. 


\section{Measurement of $k_{\mathbf{G}}$}

In this study, it was observed that the $\mathrm{CO}_{2}$ concentration in the headspace of shake culture in the Erlenmeyer and Sakaguchi flasks was very high compared with that in the atmosphere. Consequently, we measured $\mathrm{CO}_{2}$ concentration in the headspace of flasks with shaking in a nonsteady state by using the CDMSS. $k_{\mathrm{G}}$, an indicator of $\mathrm{CO}_{2}$ ventilation capacity, was calculated as follows:

$$
-\ln \frac{C_{\min }^{\prime}-C_{\prime}^{\prime}}{C_{\min }^{\prime}-C_{0}^{\prime}}=k_{\mathrm{G}} \cdot t
$$

where $t$ is time (s), $\mathrm{C}_{0}^{\prime}$ is the dissolved $\mathrm{CO}_{2}$ concentration at $t=0(\mathrm{mg} / \mathrm{L})$, and $\mathrm{C}_{\min }^{\prime}$ is the minimum $\mathrm{CO}_{2}$ concentration in fresh air. The minimum $\mathrm{CO}_{2}$ concentration, a very important factor for Eq. 2, was determined just before measurements were taken under all conditions.

Under all conditions, we obtained data until $\mathrm{CO}_{2}$ concentration decreased from 3.5 to $0.5 \%$. When the $R^{2}$ value of the approximate expression of Eq. 2 exceeded 0.990, the slope of the graph was set as $k_{\mathrm{G}}$. $\mathrm{CO}_{2}$ was added to the headspace of each flask via breathable culture stoppers until its level reached at least $5 \%$. Shaking was initiated and after the $\mathrm{CO}_{2}$ concentration in the headspace had decreased to $3.5 \%$, headspace $\mathrm{CO}_{2}$ concentration was measured every $20 \mathrm{~s}$.

\section{Measurement of culture factors}

The $\mathrm{UOD}_{660}$ (unit optical density at $660 \mathrm{~nm}$ ) and $\mathrm{pH}$ of the culture broth were measured, which was sampled without interrupted shaking by CDMSS, using V-570 spectrophotometer (JASCO, Tokyo, Japan) and $\mathrm{pH}$ meter (HORIBA, Kyoto, Japan), respectively. To ensure minimal decrease in the volume of the culture broth owing to sampling from the same flask, the total sampling volume was maintained at $<10 \%$ of the total amount of the initial culture medium. All measurements were performed in duplicates.

\section{Monitoring of $\mathrm{CO}_{2}$ and $\mathrm{O}_{2}$ concentration in shake-culture flasks}

In our previous study, the concentrations of $\mathrm{CO}_{2}$ and $\mathrm{O}_{2}$ in the gas-liquid phases were monitored using CDMSS in shake culture (Takahashi et al. 2017). In this study, CDMSS was used to monitor the headspace and the dissolved gases in various shake-culture flasks. The results are expressed in mean in Figs. 1 and 4, and were confirmed to be highly reproducible as in the case of development of CDMSS (Takahashi et al. 2017). A gaseous gradient tends to form in the headspace of the flask when microorganisms present under rich nutrient sources. In

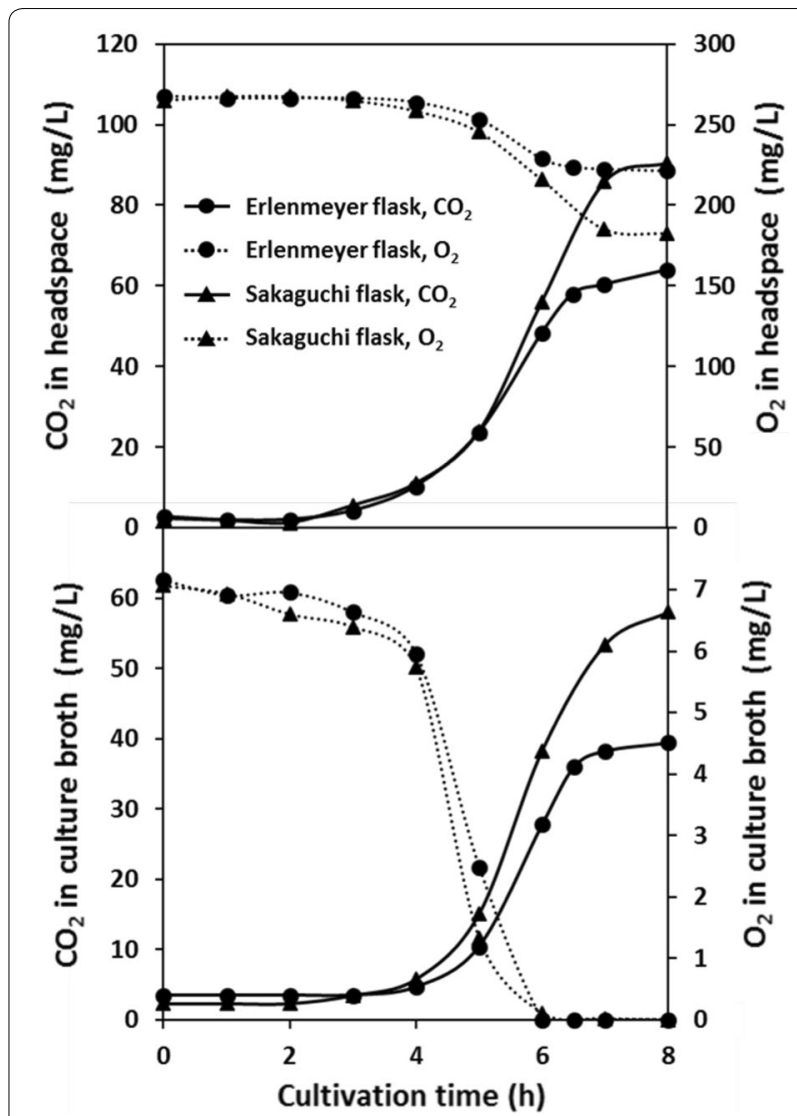

Fig. 1 Real-time $\mathrm{CO}_{2}$ and $\mathrm{O}_{2}$ concentrations in conventional flasks in shake culture of E. coli K12 IFO3301. Shake-flask culture conditions for both flasks were set as $100 \mathrm{~mL}$ of $\mathrm{LB}$ medium at $30^{\circ} \mathrm{C}$, and 200 rpm in the case of Erlenmeyer flask and 120 strokes/min shaking frequency and $70 \mathrm{~mm}$ shaking diameter in the case of Sakaguchi flask. Both 500-mL Erlenmeyer and Sakaguchi flasks were equipped with breathable culture stoppers and CDMSS

this study, $\mathrm{CO}_{2}$ and $\mathrm{O}_{2}$ in the flask gas phase were monitored under well-mixed conditions by gaseous circulation using CDMSS. No sedimentation or clogging of the cells was observed in the circulation system of shake flask culture.

\section{Results}

At the first, we compared typical shake flask cultures of E. coli $\mathrm{K} 12$ IFO3301 performed with Erlenmeyer and Sakaguchi flasks. E. coli K12 IFO3301 shake culture in Erlenmeyer and Sakaguchi flasks with CDMSS resulted in similar dissolved oxygen concentration (Fig. 1). E. coli K12 IFO3301 growth was gradual for 2 to $4 \mathrm{~h}$ and then exponential from 4 to $7 \mathrm{~h}$ (Fig. 2). The dissolved oxygen concentration gradually decreased till $4 \mathrm{~h}$, then declined sharply and was depleted by $6 \mathrm{~h}$ (Fig. 1). Although the 


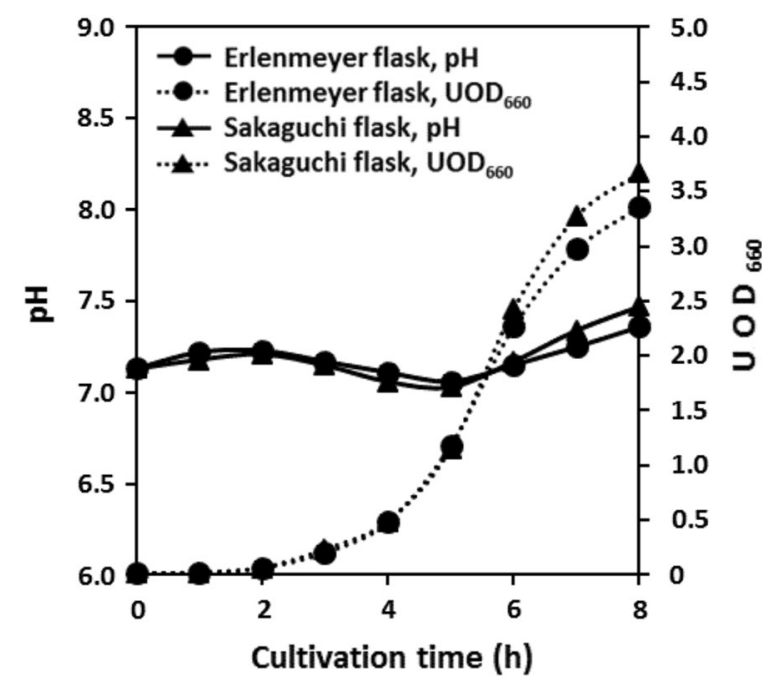

Fig. 2 Changes in culture factors in the conventional shake-flask cultures

$\mathrm{O}_{2}$ concentration in the headspace was not depleted, it decreased in parallel with the decrease in dissolved oxygen concentration. The $\mathrm{CO}_{2}$ concentration in the headspace and culture broth increased as the $\mathrm{O}_{2}$ concentration decreased (Fig. 1). There was almost no significant difference in $\mathrm{pH}$, cell growth, or dissolved oxygen concentration between the cultures in the Erlenmeyer and Sakaguchi flasks (Figs. 1 and 2). In Erlenmeyer and Sakaguchi flasks, E. coli K12 IFO3301 grew to some extent after the dissolved oxygen was depleted. Further, it plateaued, suggesting that $\mathrm{O}_{2}$ availability was rate limiting for growth (Figs. 1 and 2). Even though concentration of $\mathrm{CO}_{2}$ in the culture broth differed between the Erlenmeyer and Sakaguchi flasks, almost no difference in $\mathrm{pH}$ or growth was observed. In the conventional shake-flask culture, very large $\mathrm{CO}_{2}$ accumulations were observed in the gas and liquid phases compared to the atmosphere condition. The maximum $\mathrm{CO}_{2}$ concentration in culture broth and headspace was $40 \mathrm{mg} / \mathrm{L}$ and $63 \mathrm{mg} / \mathrm{L}$ in Erlenmeyer and $60 \mathrm{mg} / \mathrm{L}$ and $91 \mathrm{mg} / \mathrm{L}$ in Sakaguchi flasks, respectively (Fig. 1).

Oxygen supply capacity and ventilation capacity were quantified for comparison in different flasks and shaking conditions. The $k_{\mathrm{L}} a$ and $k_{\mathrm{G}}$ values measured in various shake-culture flasks with CDMSS are shown in Fig. 3. The time course of E. coli K12 IFO3301 shake culture is also presented in Figs. 4 and 5 by using cylindrical flask and baffled cylindrical flask. E. coli K12 IFO3301 grew at a similar rate in all the flasks for up to $5 \mathrm{~h}$, at which point, growth reached a steady state in the cylindrical flask and increased in the other flasks (Figs. 2, 5). The measured maximum $\mathrm{UOD}_{660}$ decreased

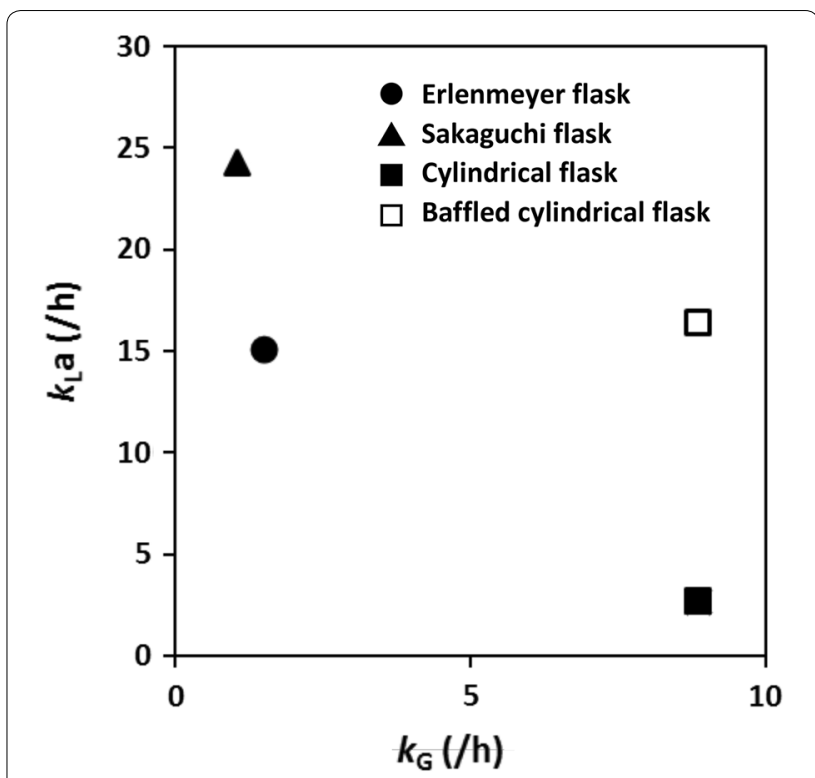

Fig. 3 Relationship between $k_{L}$ a and $k_{\mathrm{G}}$ in various shake flasks

in the following order: baffled cylindrical flask $=4.70$, Sakaguchi flask $=3.67$, Erlenmeyer flask $=3.36$, cylindrical flask $=2.25$ (Figs. 2, 5). The dissolved oxygen concentration decreased similarly in all the flasks and was depleted after $6 \mathrm{~h}$ (Figs. 1, 4). The $\mathrm{O}_{2}$ concentration in the headspace of the Erlenmeyer and Sakaguchi flasks decreased, but it remained the same as its initial value in the cylindrical flask and baffled cylindrical flask. Cylindrical flask and baffled cylindrical flask maintained very low $\mathrm{CO}_{2}$ concentrations in the headspace and culture broth compared with Erlenmeyer and Sakaguchi flasks (Figs. 1,4). The pH of the culture broth was the same in all the flasks during the first half of the shake culture but increased after $5 \mathrm{~h}$ in the cylindrical flask and decreased after $7 \mathrm{~h}$ in the baffled cylindrical flask, compared with those in the Erlenmeyer and Sakaguchi flasks (Fig. 5). In all the shake-culture flasks, the growth course of E. coli K12 IFO3301 cannot be explained only by $k_{\mathrm{L}} a$. For example, the Sakaguchi flask-with a low $k_{\mathrm{G}}$ and a high $k_{\mathrm{L}} a$-was slightly superior to the Erlenmeyer flask in terms of cell growth (Fig. 3), as the $k_{\mathrm{L}}$ a of the Sakaguchi flask was 1.61 times that of the Erlenmeyer flask ([ $k_{\mathrm{L}}$ a of Sakaguchi flask: 24.3/ $k_{\mathrm{L}}$ a of Erlenmeyer flask: 15.1$\left.]\right)$ and the maximum growth in the Sakaguchi flask was 1.1 times that in the Erlenmeyer flask ([UOD 660 of Sakaguchi flask: $3.67 /$ $\mathrm{UOD}_{660}$ of Erlenmeyer flask: 3.36$\left.]\right)$. The maximum cell growth rate, $k_{\mathrm{G}}$, and $k_{\mathrm{L}}$ a in baffled cylindrical flask were 2 times higher (4.7 in baffled cylindrical flask/2.3 in cylindrical flask), identical, and 5.9 times higher (16.4 


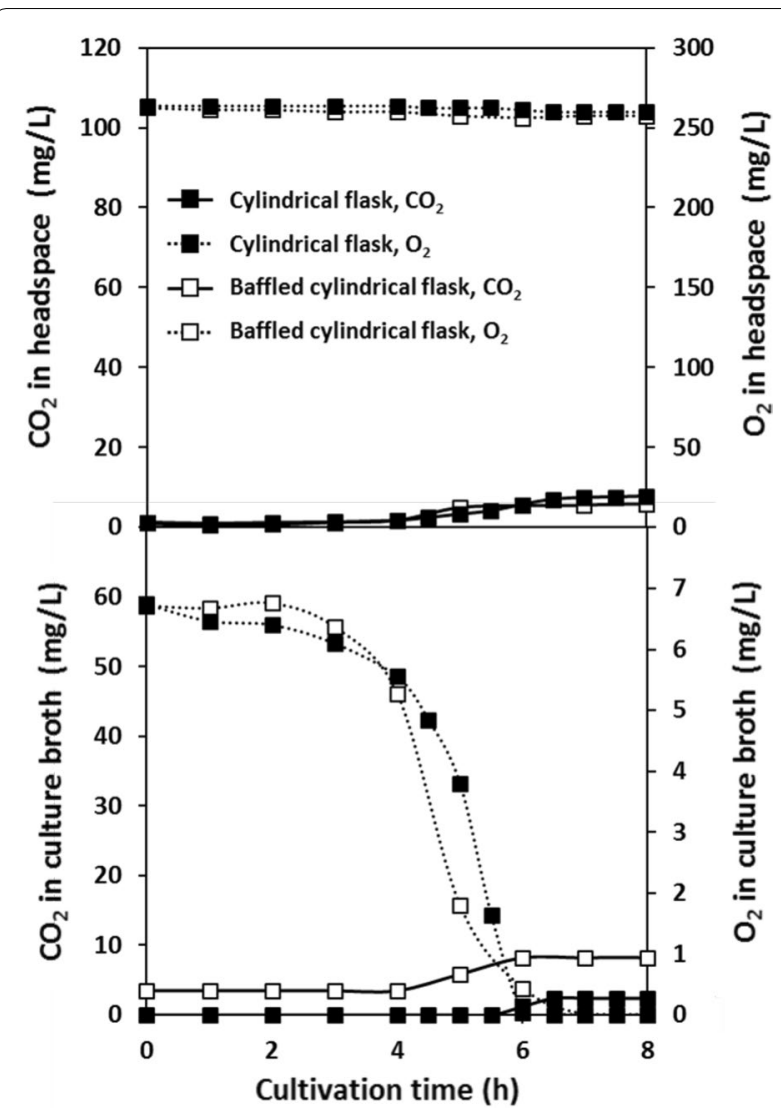

Fig. 4 Real-time $\mathrm{CO}_{2}$ and $\mathrm{O}_{2}$ concentrations in cylindrical flasks during shaking culture of $E$. coli K12 IFO3301. Shake-flask culture conditions were 100 and $50 \mathrm{~mL}$ of LB medium at $30^{\circ} \mathrm{C}, 200 \mathrm{rpm}$ with $70 \mathrm{~mm}$ shaking diameter, and $500-\mathrm{mL}$ cylindrical flask without baffle and 500-mL baffled cylindrical flask, both equipped with breathable culture stoppers and CDMSS

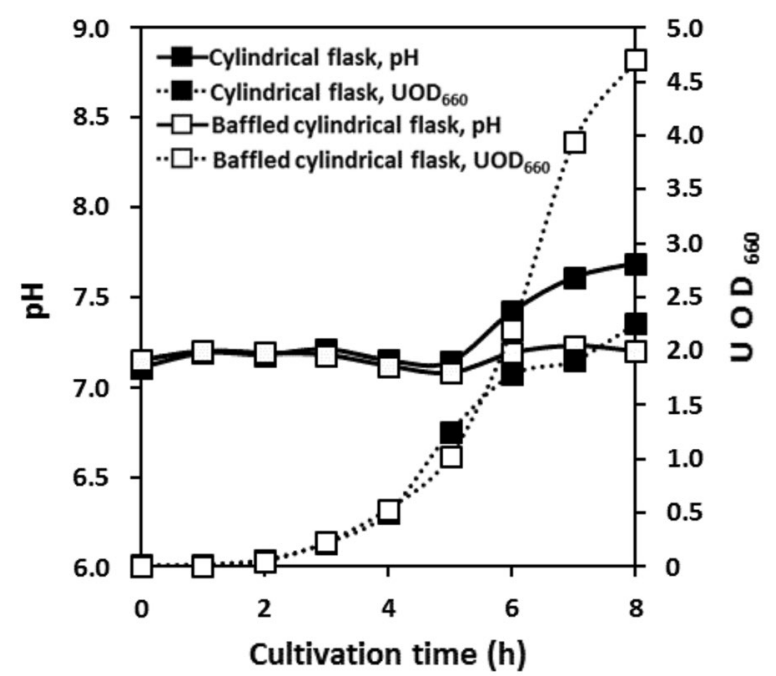

Fig. 5 Changes in culture factors in shake culture using cylindrical flask and baffled cylindrical flask in baffled cylindrical flask/2.8 in cylindrical flask) than those in the cylindrical flask, respectively.

\section{Discussion}

We used Erlenmeyer (rotary shaker with $70 \mathrm{~mm}$ shaking diameter at $200 \mathrm{rpm}$ ) and Sakaguchi (reciprocating shaker with $70 \mathrm{~mm}$ shaking diameter at 120 strokes/min) flasks for shaking conditions of E. coli K12 IFO3301 cultivation. These shaking conditions are standard about batch culture on flask scale. It was suggested that the logphase growth of E. coli K12 IFO3301 can be predicted by monitoring $\mathrm{CO}_{2}$ in the headspace of conventional shake flasks, because the $\mathrm{CO}_{2}$ concentration and the growth of E. coli K12 IFO3301 showed a very good correlation. Similar data have been reported by using a fluorescent sensor (Ge and Rao 2012) and CDMSS (Takahashi et al. 2017) in shaking culture by Erlenmeyer flask. These phenomena occur when the $\mathrm{CO}_{2}$ due to the respiratory activity of $E$. coli begins to fill the flask gas phase. However, in the cylindrical flask that could not supply enough oxygen, E. coli K12 IFO3301 did not grow much and the $\mathrm{CO}_{2}$ concentration remained almost unchanged. In the case of very low respiratory activity and need for detailed data, highly sensitive monitoring is required such as RAMOS.

We have an approach about shake-flask culture that goes beyond monitoring. It is possible that conventional shake culture using Erlenmeyer and Sakaguchi flasks has not previously taken into consideration the effect of $\mathrm{CO}_{2}$ accumulation in aerobic culture. We reported that E. coli growth improves in shake culture in Erlenmeyer and Sakaguchi flasks when the $\mathrm{CO}_{2}$ of flask gas phase, which accumulates due to respiratory activity, is maintained at low concentration by CDMSS with gaseous $\mathrm{CO}_{2}$ adsorbent (Takahashi et al. 2017; Takahashi and Aoyagi 2018c). This supports the idea that $\mathrm{CO}_{2}$ concentration in flask headspace has a significant impact on culture growth rate. This study compares different flasks by quantifying ventilation capacity and proves the above idea. Despite the almost same $k_{\mathrm{L}} a$ in both flasks, the $k_{\mathrm{G}}$ ratio (Baffled cylindrical flask/Erlenmeyer flask) was 6.4 (8.9/1.4) and the maximum growth ratio (Baffled cylindrical flask/Erlenmeyer flask) was 1.4 (4.7/3.4). The $\mathrm{CO}_{2}$ concentration in baffled cylindrical flask and cylindrical flask was identical, despite the former supporting higher cell growth. This may be due to not only lower amount of medium and larger headspace but also sufficient ventilation in the baffled cylindrical flask. It is true that $E$. coli K12 IFO3301 growth requires a high $k_{\mathrm{L}} a$, but we conclude that not only high $k_{\mathrm{L}} a$ but also high $k_{\mathrm{G}}$ is required by comparison of various flasks. It was noted that the detailed monitoring of flask gas phase in shaking culture became difficult in the case of the flask with high ventilation capacity. 
$\mathrm{CO}_{2}$ has been reported to have a significant impact on culture growth (Blombach and Takors 2015, Takahashi and Aoyagi 2018a). Furthermore, $\mathrm{CO}_{2}$ concentration in the headspace can be maintained at low levels by adding adsorbent to the bypass part of CDMSS (Takahashi et al. 2017; Takahashi and Aoyagi 2018c). However, it may be difficult to set monitoring devices and adsorbent to multiple shake flasks when screening for secondary metabolites and developing early-stage bioprocesses for microorganisms (e.g., when examining culture conditions). In this study, we used baffled cylindrical flask, which has a headspace environment with a low $\mathrm{CO}_{2}$ accumulation without using $\mathrm{CO}_{2}$ adsorbent. However, the handling of flask set on the shaking table was the same as that of the conventional flask, but when the medium volume was $100 \mathrm{~mL}$ without baffled, the oxygen supply capacity was significantly reduced due to the lack of formation of thin water film on the flask wall. Hence, we ensured that $k_{\mathrm{L}}$ a increased by inserting a baffle and lower working volume, and allowing the culture broth that climbed up the wall of the flask to hit the baffle during rotary. At that time, the working volume was also changed, but the oxygen supply capacity was quantified so that comparison was possible even under different flask conditions. Therefore, we expect that redesign of flasks based on not only $k_{\mathrm{L}}$ a but also $k_{\mathrm{G}}$ (as shown in Fig. 3) may contribute to a wide range of fields employing cell culture.

\section{Abbreviations}

CDMSS: Circulation direct monitoring and sampling system; RAMOS: Respiration Activity Monitoring System; $\cup D_{660}$ : Unit optical density at $660 \mathrm{~nm}$.

\section{Acknowledgements}

The manuscript has been edited carefully by native-English-speaking professional editor from Editage, a division of Cactus Communications.

\section{Authors' contributions}

MT and HA designed the research. HA supervised the research. MT created the experimental design and performed all experiments. MT and HA analysed the data. MT and HA wrote the manuscript. Both authors read and approved the final manuscript.

\section{Funding}

This work was supported by Noda Institute for Scientific Research Young Investigator Research Grant and Japan Society for the Promotion Science (JSPS) KAKENHI Grant-in-Aid for Early-Career Scientists [20K15097] (grants to Masato Takahashi.). This work was also supported in part by JSPS KAKENHI Grant-in Aid for Scientific Research B (19H03086), the Sumitomo Electric Industries Group Corporate Social Responsibility Foundation, and Noda Institute for Scientific Research Grant (grants to Hideki Aoyagi).

\section{Ethics approval and consent to participate}

Not applicable.

\section{Consent for publication}

Not applicable.

\section{Competing interests}

The authors declare that they have no competing interests.

Received: 16 March 2020 Accepted: 9 April 2020

Published online: 19 April 2020

\section{References}

Anderlei T, Zang W, Papaspyrou M, Büchs J (2004) Online respiration activity measurement (OTR, CTR, RQ) in shake flasks. Biochem Eng J 17:187-194

Blombach B, Takors R (2015) $\mathrm{CO}_{2}$-intrinsic product, essential substrate, and regulatory trigger of microbial and mammalian production processes. Front Bioeng Biotechnol 3:108

Eibl R, Meier P, Stutz I, Schildberger D, Hühn T, Eibl D (2018) Plant cell culture technology in the cosmetics and food industries: current state and future trends. Appl Microbiol Biotechnol 102:8661-8675

Ge X, Rao G (2012) Real-time monitoring of shake flask fermentation and off gas using triple disposable noninvasive optical sensors. Biotechnol Prog 28:872-877

Hirasawa T, Nakakura Y, Yoshikawa K, Ashitani K, Nagahisa K, Furusawa C, Katakura Y, Shimizu H, Shioya S (2006) Comparative analysis of transcriptional responses to saline stress in the laboratory and brewing strains of Saccharomyces cerevisiae with DNA microarray. Appl Microbiol Biotechnol 70:346-357

Klöckner W, Büchs J (2012) Advances in shaking technologies. Trends Biotechnol 30:307-314

Kluyver A, Perquin L (1933) Zur methodik der schimmel-stoffwechseluntersuchung. Biochem Z 266:68-81 (in German)

Matsuda F, Kinoshita S, Nishino S, Tomita A, Shimizu H (2017) Targeted proteome analysis of single-gene deletion strains of Saccharomyces cerevisiae lacking enzymes in the central carbon metabolism. PLoS ONE 12:e0172742

Nojiri M, Hibi M, Shizawa H, Horinouchi N, Yasohara Y, Takahashi S, Ogawa J (2015) Imidase catalyzing desymmetric imide hydrolysis forming optically active 3-substituted glutaric acid monoamides for the synthesis of gamma-aminobutyric acid (GABA) analogs. Appl Microbiol Biotechnol 99:9961-9969

Omura S, Iwai Y, Hirano A, Nakagawa A, Awaya J, Tsuchya H, Takahashi Y, Masuma R (1977) A new alkaloid AM-2282 OF Streptomyces origin taxonomy, fermentation, isolation and preliminary characterization. J Antibiot 30:275-282

Platas OB, Sandig V, Pörtner R, Zeng AP (2013) Evaluation of process parameters in shake flasks for mammalian cell culture. BMC Proc 7:P17

Reynoso-Cereceda GI, Garcia-Cabrera RI, Valdez-Cruz NA, Trujillo-Roldán MA (2016) Shaken flasks by resonant acoustic mixing versus orbital mixing: mass transfer coefficient $k_{\mathrm{L}}$ a characterization and Escherichia coli cultures comparison. Biochem Eng J 105:379-390

Schulte A, Schilling JV, Nolten J, Korona A, Krömke H, Vennekotter JB, Schillheim B, Wessling M, Conrath U, Büchs J (2018) Parallel online determination of ethylene release rate by Shaken Parsley cell cultures using a modified RAMOS device. BMC Plant Biol 18:101

Shiota H, Sakaguchi KI (1950) Studies on the production of mold protease in the submerged culture part I. Nippon Nogeikagaku Kaishi 23:426-429 (in Japanese)

Takahashi M, Aoyagi H (2018a) Effect of intermittent opening of breathable culture plugs and aeration of headspace on the structure of microbial communities in shake-flask culture. J Biosci Bioeng 126:96-101

Takahashi M, Aoyagi H (2018b) Practices of shake-flask culture and advances in monitoring $\mathrm{CO}_{2}$ and $\mathrm{O}_{2}$. Appl Microbiol Biotechnol 102:4279-4289

Takahashi M, Aoyagi $\mathrm{H}$ (2018c) Monitoring of $\mathrm{CO}_{2}$ and $\mathrm{O}_{2}$ concentrations in the headspace of Sakaguchi flasks during liquid culture of microorganism. Appl Microbiol Biotechnol 102:6637-6645

Takahashi M, Sawada Y, Aoyagi H (2017) Development of a circulation direct sampling and monitoring system for $\mathrm{O}_{2}$ and $\mathrm{CO}_{2}$ concentrations in the gas-liquid phases of shake-flask systems during microbial cell culture. AMB Express 7:163

Weirether FJ, Walker JS, Lincoln RE (1968) A precise method for replicating suspension cultures of mammalian cells. Appl Microbiol 16:841-844

Wewetzer SJ, Kunze M, Ladner T, Luchterhand B, Roth S, Rahmen N, Kloß R, Costa E, Silva A, Regestein L, Büchs J (2015) Parallel use of shake flask and microtiter plate online measuring devices (RAMOS and BioLector) reduces the number of experiments in laboratory-scale stirred tank bioreactors. J Biol Eng 9:9

\section{Publisher's Note}

Springer Nature remains neutral with regard to jurisdictional claims in published maps and institutional affiliations. 